\title{
Binding effects in proton-nucleus elastic scattering
}

\author{
R. Crespo \\ Departamento de Física, Instituto Superior Técnico, Avenida Rovisco Pais, 1096 Lisboa, Portugal \\ and Centro de Física Nuclear, Universidade de Lisboa, Avenida Prof. Gama Pinto 2, \\ 1699 Lisboa, Portugal \\ R. C. Johnson and J. A. Tostevin \\ Department of Physics, University of Surrey, Guildford, Surrey, GU2 5XH, United Kingdom
}

(Received 26 January 1993)

\begin{abstract}
The effects of the struck nucleon-core binding potential on the first-order term of the Kerman, McManus, and Thaler (KMT) expansion of the nucleon-nucleus optical potential are calculated for the elastic scattering of protons from ${ }^{16} \mathrm{O}$ at $200 \mathrm{MeV}$. The binding effects are expressed as a momentum-transfer-dependent energy shift in the off-shell nucleon-nucleon $t$ matrix. Numerical results are based on the Paris nucleon-nucleon potential together with both harmonic oscillator or Woods-Saxon single-particle wave functions for the target. The calculations show that these binding effects introduce only small corrections to the optical potential and calculated observables in the intermediate energy region when compared with the impulse approximation.
\end{abstract}

PACS number(s): $25.40 . \mathrm{Cm}, 24.10 .-\mathrm{i}, 24.10 . \mathrm{Ht}, 24.70 .+\mathrm{s}$

\section{INTRODUCTION}

A common feature of many approaches to the firstorder term of the Kerman, McManus, and Thaler (KMT) [1] expansion of the optical potential is the use of the impulse approximation. At intermediate energies it is assumed the incident energy is sufficiently large that the effects of the nuclear medium, specifically the mean field potential binding the struck nucleon to the core of target nucleons and the struck nucleon binding energy, can be neglected [2-4]. The collision of the incident and struck nucleons is thus considered free. An important practical consequence of this approximation is that momentum conservation within the $N N$ collision can be assumed. The Pauli blocking medium effects, associated with the second-order terms of the KMT potential, have recently been evaluated [5]. However, all calculations are far from satisfactory in their description of the available experimental data, in particular with regard to the reactive content of the calculated optical potential. In this work we investigate the struck nucleon-core binding effects upon the first-order KMT optical potential.

An intrinsic difficulty in including such binding effects is that they require the solution of a three-body problem $[6,7]$. Several attempts to include this effect in the case of pion-nucleus scattering can be found in the literature. They have lead to some contradictory results; see, for example, the review of Thomas and Landau and references therein [8]. Two attempts have previously been made to study the validity of the impulse approximation in the evaluation of the first-order KMT optical potential for proton scattering $[2,9]$. In the first [2] only the binding energy of the struck nucleon was considered, however, a detailed treatment of the momenta of the interacting nucleon pair and hence an explicit inclusion of the variation of the energy of the $N N$ transition amplitude was included. This binding energy correction leads to an overall negative shift in the energy parameter in the $N N$ amplitude. We note particularly that the inclusion of this negative energy shift was largely responsible for the significant corrections to the impulse approximation reported in that work. In the second work [9] both the binding energy and the binding potential were considered through the use of an effective mass approach. In both these works the ability of the binding potential to actually induce momentum transfers in the nucleon-nucleus collision is ignored. It is this effect, together with the binding energy effect of Ref. [2] which is carefully considered here. The nonlocality of the binding potential is ignored. Throughout we use the off-shell $N N$ transition matrix derived from the Paris potential [10].

\section{THE OPTICAL POTENTIAL}

The first-order term of the KMT nucleon-nucleus optical potential is given by the expression $[1,5]$

$$
U=\frac{A-1}{A} \sum_{\alpha}\left\langle\alpha\left|\hat{t}_{01}\left(\hat{\omega}_{\alpha}\right)\right| \alpha\right\rangle
$$

where we have assumed that the target ground state is described by a single Slater determinant of occupied singleparticle wave functions $|\alpha\rangle$ with energy eigenvalues $\epsilon_{\alpha}$, and the sum in Eq. (1) runs over all occupied states. Here the $N N$ transition operator $\hat{t}_{01}\left(\hat{\omega}_{\alpha}\right)$ is a three-body operator and satisfies the integral equation

$$
\hat{t}_{01}\left(\hat{\omega}_{\alpha}\right)=v_{01}+v_{01} \hat{g}\left(\hat{\omega}_{\alpha}\right) \hat{t}_{01}\left(\hat{\omega}_{\alpha}\right),
$$

where $v_{01}$ is the free $N N$ interaction. The propagator is 


$$
\hat{g}\left(\hat{\omega}_{\alpha}\right)=\frac{1}{\hat{\omega}_{\alpha}^{+}-K_{0}-K_{1}-V_{1}},
$$

with $K_{0}\left(K_{1}\right)$ the kinetic energy operator for the projectile (struck) nucleon, and $V_{1}$ the struck nucleon-core binding potential. The transition operator has energy parameter $\hat{\omega}_{\alpha}=E+\epsilon_{\alpha}$ with $E$ the incident nucleon energy in the nucleon-target center-of-mass frame.

To reduce the evaluation of the optical potential to a two-body problem we introduce the free $N N$ transition amplitude $t_{01}\left(\omega_{\alpha}\right)$, satisfying the integral equation

$$
t_{01}\left(\omega_{\alpha}\right)=v_{01}+v_{01} g\left(\omega_{\alpha}\right) t_{01}\left(\omega_{\alpha}\right)
$$

with $g\left(\omega_{\alpha}\right)$ the free $N N$ propagator given by

$$
g\left(\omega_{\alpha}\right)=\frac{1}{\omega_{\alpha}^{+}-K_{0}-K_{1}} .
$$

The energy parameter is now written $\omega_{\alpha}=E+\mathcal{E}_{\alpha}$, where in the following the effective single-particle energies $\mathcal{E}_{\alpha}$ will be chosen to cancel the leading binding potential correction to the two-body transition amplitude. The two $N N$ transition amplitudes are related through the exact relationship

$$
\begin{aligned}
\hat{t}_{01}\left(\hat{\omega}_{\alpha}\right)=t_{01}\left(\omega_{\alpha}\right) & +t_{01}\left(\omega_{\alpha}\right)\left[\hat{g}\left(\hat{\omega}_{\alpha}\right)-g\left(\omega_{\alpha}\right)\right] \hat{t}_{01}\left(\hat{\omega}_{\alpha}\right) \\
=t_{01}\left(\omega_{\alpha}\right) & +t_{01}\left(\omega_{\alpha}\right) g\left(\omega_{\alpha}\right)\left[\omega_{\alpha}-\hat{\omega}_{\alpha}+V_{1}\right] \\
& \times g\left(\omega_{\alpha}\right) t_{01}\left(\omega_{\alpha}\right)+\cdots .
\end{aligned}
$$

In the momentum space representation, the matrix elements of the optical potential are

$$
\begin{aligned}
\left\langle\mathbf{k}_{0}^{\prime}|U| \mathbf{k}_{0}\right\rangle= & \frac{A-1}{A} \sum_{\alpha}\left\langle\mathbf{k}_{0}^{\prime} \alpha\left|\hat{t}_{01}\left(\hat{\omega}_{\alpha}\right)\right| \alpha \mathbf{k}_{0}\right\rangle \\
= & \frac{A-1}{A} \sum_{\alpha}\left\langle\mathbf{k}_{0}^{\prime} \alpha\left|t_{01}\left(\omega_{\alpha}\right)\right| \alpha \mathbf{k}_{0}\right\rangle \\
& +\left\langle\mathbf{k}_{0}^{\prime}|\Delta U| \mathbf{k}_{0}\right\rangle,
\end{aligned}
$$

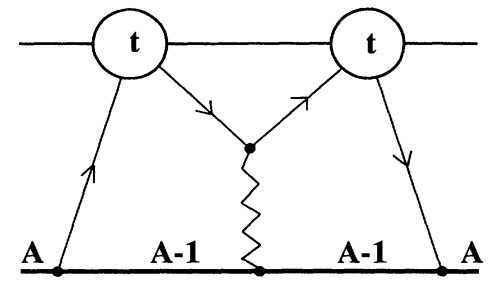

FIG. 1. Diagrammatic representation of leading correction to the optical potential to first order in the binding potential, indicated in the figure by the zig-zag line.

where $\mathbf{k}_{0}$ and $\mathbf{k}_{0}^{\prime}$ are projectile momenta in the nucleonnucleus $(N A)$ center-of-mass frame. The leading binding potential correction to the two-body amplitude is therefore

$$
\begin{aligned}
\left\langle\mathbf{k}_{0}^{\prime}|\Delta U| \mathbf{k}_{0}\right\rangle=\frac{A-1}{A} \sum_{\alpha} & \left\langle\mathbf{k}_{0}^{\prime} \alpha\right| t_{01}\left(\omega_{\alpha}\right) g\left(\omega_{\alpha}\right) \\
& \times \xi_{\alpha} g\left(\omega_{\alpha}\right) t_{01}\left(\omega_{\alpha}\right)\left|\alpha \mathbf{k}_{0}\right\rangle
\end{aligned}
$$

with $\xi_{\alpha}=\left[\omega_{\alpha}-\hat{\omega}_{\alpha}+V_{1}\right]$. The amplitude, represented schematically in Fig. 1, involves a double scattering between the projectile and struck nucleon. Between scatterings the struck nucleon does not conserve its momentum due to the interaction $V_{1}$ with the remaining target nucleons, represented by the zigzag line in the figure. Only a single scattering between the struck nucleon and the core of nucleons is included.

Introducing unit partitions, in momentum space, for the incident and struck nucleons then

$$
\begin{aligned}
\left\langle\mathbf{k}_{0}^{\prime}|\Delta U| \mathbf{k}_{0}\right\rangle=\frac{A-1}{A} \sum_{\alpha} \int & d \mathbf{k}_{1} d \mathbf{k}_{1}^{\prime} d \mathbf{k}_{0}^{\prime \prime} d \mathbf{k}_{1}^{\prime \prime} d \mathbf{k}_{1}^{\prime \prime \prime} \phi_{\alpha}^{*}\left(\mathbf{k}_{1}^{\prime}\right)\left\langle\mathbf{k}_{0}^{\prime} \mathbf{k}_{1}^{\prime}\left|t_{01}\left(\omega_{\alpha}\right)\right| \mathbf{k}_{0}^{\prime \prime} \mathbf{k}_{1}^{\prime \prime}\right\rangle g\left(\omega_{\alpha}, k_{0}^{\prime \prime}, k_{1}^{\prime \prime}\right)\left\langle\mathbf{k}_{1}^{\prime \prime}\left|\xi_{\alpha}\right| \mathbf{k}_{1}^{\prime \prime \prime}\right\rangle g\left(\omega_{\alpha}, k_{0}^{\prime \prime}, k_{1}^{\prime \prime \prime}\right) \\
& \times\left\langle\mathbf{k}_{0}^{\prime \prime} \mathbf{k}_{1}^{\prime \prime \prime}\left|t_{01}\left(\omega_{\alpha}\right)\right| \mathbf{k}_{0} \mathbf{k}_{1}\right\rangle \phi_{\alpha}\left(\mathbf{k}_{1}\right)
\end{aligned}
$$

where the propagators are

$$
g\left(\omega_{\alpha}, k_{0}^{\prime \prime}, k_{1}^{\prime \prime}\right)=\left[\omega_{\alpha}^{+}-\frac{\hbar^{2} k_{0}^{\prime 2}}{2 m}-\frac{\hbar^{2} k_{1}^{\prime 2}}{2 m}\right]^{-1}
$$

Applying momentum conservation at each of the free $N N$ vertices, now gives, e.g.,

$$
\begin{aligned}
\left\langle\mathbf{k}_{0}^{\prime} \mathbf{k}_{1}^{\prime}\left|t_{01}\left(\omega_{\alpha}\right)\right| \mathbf{k}_{1}^{\prime \prime} \mathbf{k}_{0}^{\prime \prime}\right\rangle= & \delta\left(\mathbf{k}_{0}^{\prime}+\mathbf{k}_{1}^{\prime}-\mathbf{k}_{1}^{\prime \prime}-\mathbf{k}_{0}^{\prime \prime}\right) \\
& \times\left\langle\frac{1}{2}\left(\mathbf{k}_{0}^{\prime}-\mathbf{k}_{1}^{\prime}\right)\left|t_{01}\left(\omega_{\alpha}\right)\right|\right. \\
& \left.\times \frac{1}{2}\left(\mathbf{k}_{0}^{\prime \prime}-\mathbf{k}_{1}^{\prime \prime}\right)\right\rangle
\end{aligned}
$$

where the $N N$ amplitude corresponds to an energy parameter of

$$
\omega_{\alpha}(N N)=\omega_{\alpha}-\hbar^{2}\left(\mathbf{k}_{0}^{\prime}+\mathbf{k}_{1}^{\prime}\right)^{2} / 4 m
$$

in the $N N$ center-of-mass frame. The momentumconserving $\delta$-functions dictate that $\mathbf{k}_{1}^{\prime \prime}=\mathcal{K}=\mathbf{k}_{0}^{\prime}+\mathbf{k}_{1}^{\prime}-\mathbf{k}_{0}^{\prime \prime}$ and that $\mathbf{k}_{1}^{\prime \prime \prime}=\mathcal{K}^{\prime}=\mathbf{k}_{0}+\mathbf{k}_{1}-\mathbf{k}_{0}^{\prime \prime}$, therefore

$$
\begin{aligned}
\left\langle\mathbf{k}_{1}^{\prime \prime}\left|\xi_{\alpha}\right| \mathbf{k}_{1}^{\prime \prime \prime}\right\rangle & =\xi_{\alpha}\left(\mathbf{k}_{1}^{\prime \prime}-\mathbf{k}_{1}^{\prime \prime \prime}\right) \\
& =\xi_{\alpha}\left(\mathbf{k}_{0}^{\prime}+\mathbf{k}_{1}^{\prime}-\mathbf{k}_{0}-\mathbf{k}_{1}\right)
\end{aligned}
$$

and the correction term reads 


$$
\begin{aligned}
\left\langle\mathbf{k}_{0}^{\prime}|\Delta U| \mathbf{k}_{0}\right\rangle=\frac{A-1}{A} \sum_{\alpha} \int & d \mathbf{k}_{1} d \mathbf{k}_{1}^{\prime} d \mathbf{k}_{0}^{\prime \prime} \phi_{\alpha}^{*}\left(\mathbf{k}_{1}^{\prime}\right)\left\langle\frac{1}{2}\left(\mathbf{k}_{0}^{\prime}-\mathbf{k}_{1}^{\prime}\right)\left|t_{01}\left(\omega_{\alpha}\right)\right| \mathbf{k}_{0}^{\prime \prime}-\frac{1}{2}\left(\mathbf{k}_{0}^{\prime}+\mathbf{k}_{1}^{\prime}\right)\right\rangle \\
& \times g\left(\omega_{\alpha}, k_{0}^{\prime \prime}, \mathcal{K}\right) \xi_{\alpha}\left(\mathbf{k}_{0}^{\prime}+\mathbf{k}_{1}^{\prime}-\mathbf{k}_{0}-\mathbf{k}_{1}\right) g\left(\omega_{\alpha}, k_{0}^{\prime \prime}, \mathcal{K}^{\prime}\right) \\
& \times\left\langle\mathbf{k}_{0}^{\prime \prime}-\frac{1}{2}\left(\mathbf{k}_{0}+\mathbf{k}_{1}\right)\left|t_{01}\left(\omega_{\alpha}\right)\right| \frac{1}{2}\left(\mathbf{k}_{0}-\mathbf{k}_{1}\right)\right\rangle \phi_{\alpha}\left(\mathbf{k}_{1}\right) .
\end{aligned}
$$

We now neglect the dependences upon the momentum of the struck nucleon everywhere except that in the wave functions and in the binding potential. We thus assume that the single-particle states and potential are the most strongly dependent functions of the struck nucleon momentum. The correction term then reads

$$
\begin{aligned}
\left\langle\mathbf{k}_{0}^{\prime}|\Delta U| \mathbf{k}_{0}\right\rangle=\frac{A-1}{A} \sum_{\alpha} \chi_{\alpha}\left(\mathbf{k}_{0}^{\prime}-\mathbf{k}_{0}\right) \int & d \mathbf{k}_{0}^{\prime \prime} g\left(\omega_{\alpha}, k_{0}^{\prime \prime}, \mathcal{K}^{\prime}\right) g\left(\omega_{\alpha}, k_{0}^{\prime \prime}, \mathcal{K}\right) \\
& \times\left\langle\mathbf{k}_{0}^{\prime} / 2\left|t_{01}\left(\omega_{\alpha}\right)\right| \mathbf{k}_{0}^{\prime \prime}-\mathbf{k}_{0}^{\prime} / 2\right\rangle\left\langle\mathbf{k}_{0}^{\prime \prime}-\mathbf{k}_{0} / 2\left|t_{01}\left(\omega_{\alpha}\right)\right| \mathbf{k}_{0} / 2\right\rangle,
\end{aligned}
$$

where now $\mathcal{K}=\mathbf{k}_{0}^{\prime}-\mathbf{k}_{0}^{\prime \prime}$ and $\mathcal{K}^{\prime}=\mathbf{k}_{0}-\mathbf{k}_{0}^{\prime \prime}$.

The binding effects enter through the function

$$
\begin{aligned}
\chi_{\alpha}\left(\mathbf{k}_{0}^{\prime}-\mathbf{k}_{0}\right)= & \int d \mathbf{k}_{1} d \mathbf{k}_{1}^{\prime} \phi_{\alpha}^{*}\left(\mathbf{k}_{1}^{\prime}\right) \xi_{\alpha}\left(\mathbf{k}_{0}^{\prime}+\mathbf{k}_{1}^{\prime}-\mathbf{k}_{0}-\mathbf{k}_{1}\right) \\
& \times \phi_{\alpha}\left(\mathbf{k}_{1}\right) \\
= & \left(\omega_{\alpha}-\hat{\omega}_{\alpha}\right) \rho_{\alpha}\left(\mathbf{k}_{0}^{\prime}-\mathbf{k}_{0}\right)+V_{\alpha}\left(\mathbf{k}_{0}^{\prime}-\mathbf{k}_{0}\right),
\end{aligned}
$$

where we have defined

$$
\rho_{\alpha}(\mathbf{q})=\int d \mathbf{r} \phi_{\alpha}^{*}(\mathbf{r}) \exp (-i \mathbf{q} \cdot \mathbf{r}) \phi_{\alpha}(\mathbf{r})
$$

and

$$
V_{\alpha}(\mathbf{q})=\int d \mathbf{r} \phi_{\alpha}^{*}(\mathbf{r}) \exp (-i \mathbf{q} \cdot \mathbf{r}) V_{1}(r) \phi_{\alpha}(\mathbf{r})
$$

We will now assume that the target is a closed shell/subshell nucleus. We may then perform the sums over states internal to each subshell (denoted in the following by $\alpha$ ) and replace $\chi_{\alpha}\left(\mathbf{k}_{0}^{\prime}-\mathbf{k}_{0}\right)$ in Eq. (15) by

$$
\bar{\chi}_{\alpha}(q)=\left(\omega_{\alpha}-\hat{\omega}_{\alpha}\right) \bar{\rho}_{\alpha}(q)+\bar{V}_{\alpha}(q),
$$

where $\bar{\rho}_{\alpha}(q)$ is now the density of the subshell $\alpha$, i.e.,

$$
\bar{\rho}_{\alpha}(q)=\sum_{i \in \alpha} \int d \mathbf{r} \phi_{i}^{*}(\mathbf{r}) \exp (-i \mathbf{q} \cdot \mathbf{r}) \phi_{i}(\mathbf{r})
$$

and the binding potential correction $\bar{V}_{\alpha}(q)$ is similarly

$$
\bar{V}_{\alpha}(q)=\sum_{i \in \alpha} \int d \mathbf{r} \phi_{i}^{*}(\mathbf{r}) \exp (-i \mathbf{q} \cdot \mathbf{r}) V_{1}(r) \phi_{i}(\mathbf{r})
$$

with $q$ the momentum transfer $\mathbf{q}=\mathbf{k}_{0}^{\prime}-\mathbf{k}_{0}$. In order to minimize the correction to the two-body amplitude it follows from Eq. (19) that we should choose the energy parameter $\omega_{\alpha}$ such that

$$
\begin{aligned}
\omega_{\alpha}=E+\mathcal{E}_{\alpha} & =\hat{\omega}_{\alpha}-\bar{V}_{\alpha}(q) / \bar{\rho}_{\alpha}(q) \\
& =E+\epsilon_{\alpha}-\bar{V}_{\alpha}(q) / \bar{\rho}_{\alpha}(q)
\end{aligned}
$$

in which case $\left\langle\mathbf{k}_{0}^{\prime}|\Delta U| \mathbf{k}_{0}\right\rangle$ vanishes.

For ease of interpretation, the binding correction can therefore be expressed in terms of momentum-transfer- dependent effective single-particle energies given by

$$
\mathcal{E}_{\alpha}(q)=\epsilon_{\alpha}-\bar{V}_{\alpha}(q) / \bar{\rho}_{\alpha}(q),
$$

with explicit contributions from both the binding energy and the binding potential.

In the description of the target nucleus we shall not distinguish between protons and neutrons. We also fix, in the evaluation of the energy parameter of the $N N$ transition amplitude, the projectile momentum to the on-shell value $\kappa_{0}$ and therefore the amplitude is to be calculated at the energies

$$
\omega_{\alpha}(N N)=E+\mathcal{E}_{\alpha}(q)-\hbar^{2} \kappa_{0}^{2} / 4 m=E / 2+\mathcal{E}_{\alpha}(q) .
$$

Thus, in the optimal factorization approximation $[4,5]$, and displaying now the energy of the $N N$ amplitude in terms of the appropriate energy $\omega_{\alpha}(N N)$ in the $N N$ center-of-mass frame, the optical potential reads

$$
\left\langle\mathbf{k}_{0}^{\prime}|U| \mathbf{k}_{0}\right\rangle=\sum_{\alpha} \bar{\rho}_{\alpha}(q) \bar{t}_{01}\left[E / 2+\mathcal{E}_{\alpha}(q), \mathbf{q}, \mathbf{Q} / 2\right],
$$

where $\bar{t}_{01}$ is the spin-isospin average of the $N N$ transition amplitude and $\mathbf{Q}=\left(\mathbf{k}_{0}^{\prime}+\mathbf{k}_{0}\right) / 2$ [4]. In this form it is convenient to evaluate the binding correction to the optimal factorization $\omega_{\alpha}(N N)=E / 2$ as a first-order expansion in terms of the binding corrections,

$$
\begin{aligned}
\left\langle\mathbf{k}_{0}^{\prime}|U| \mathbf{k}_{0}\right\rangle= & \sum_{\alpha} \bar{\rho}_{\alpha}(q) \bar{t}_{01}(E / 2, \mathbf{q}, \mathbf{Q} / 2) \\
& +\sum_{\alpha}\left[\epsilon_{\alpha} \bar{\rho}_{\alpha}(q)-\bar{V}_{\alpha}(q)\right] \\
& \quad \times\left.\frac{\partial}{\partial \mathcal{E}} \bar{t}_{01}(\mathcal{E}, \mathbf{q}, \mathbf{Q} / 2)\right|_{\mathcal{E}=\frac{E}{2}}
\end{aligned}
$$

The second term in Eq. (26) modifies the optimal factorization approximation [4] to take into account the binding energy and potential effects. The product of the quantity $\sum_{\alpha}\left[\epsilon_{\alpha} \bar{\rho}_{\alpha}(q)-\bar{V}_{\alpha}(q)\right]$ with the partial derivative of the $N N$ transition amplitude with respect to energy, determines the relative importance of these binding effects.

\section{THE FINITE NUCLEUS MODEL}

We consider a description of the target nucleus where a shell-model single-particle potential of Woods-Saxon 
TABLE I. Woods-Saxon shell-model parameters for ${ }^{16} \mathrm{O}$.

\begin{tabular}{lcccc}
\hline \hline Shell & $\begin{array}{c}V_{0} \\
(\mathrm{MeV})\end{array}$ & $\begin{array}{c}r_{0} \\
(\mathrm{fm})\end{array}$ & $\begin{array}{c}a_{0} \\
(\mathrm{fm})\end{array}$ & $\begin{array}{c}\epsilon_{\alpha} \\
(\mathrm{MeV})\end{array}$ \\
\hline $0 P$ & 42.4 & 1.37 & 0.61 & -12.4 \\
$0 S$ & 81.5 & 1.31 & 1.66 & -44.0 \\
\hline \hline
\end{tabular}

form is assumed [5]. In determining this shell-model potential from the empirical charge density for ${ }^{16} \mathrm{O}$, we do not consider center-of-mass corrections. In practice, when using the calculated single-particle wave functions in the optical potential calculations we do not include the nucleon-core spin-orbit force and the radial eigenfunctions are taken to be independent of the isospin of the nucleon. The $0 S$ - and $0 P$-state single-particle wavefunctions $R_{n_{\alpha} \ell_{\alpha}}$ are therefore calculated as eigenfunctions of the fitted single-particle potentials but in the absence of the Coulomb and spin-orbit interactions, i.e.,

$$
\begin{array}{r}
V_{1}(r)=-V_{0} /\left\{1+\exp \left[\left(r-R_{0}\right) / a_{0}\right]\right\}, \\
R_{0}=r_{0}(A-1)^{1 / 3} .
\end{array}
$$

Table I presents the potential parameters used. The radius and diffuseness take those values obtained by fitting the experimental data $[5,11]$. The potential depths were readjusted to reproduce the $0 S$ - and $0 P$-state separation energies in the absence of the Coulomb and spin-orbit interactions.

The single-particle radial functions $R_{\alpha}(\alpha=0 S, 0 P)$, are assumed normalized to unity, i.e.,

$$
\int_{0}^{\infty} d r r^{2} R_{\alpha}(r) R_{\alpha^{\prime}}(r)=\delta_{\alpha^{\prime} \alpha}
$$

We will also consider calculations in the case of the harmonic oscillator (HO) shell model for which

$$
V_{1}(r)=\frac{1}{2} m \omega_{0}^{2} r^{2}
$$

with $\omega_{0}$ the angular frequency for the harmonic motion. For consistency with the Woods-Saxon case we will not consider center-of-mass corrections to the oscillator model. Thus, the target density distribution is evaluated directly from the $\mathrm{HO}$ single-particle wave functions using the relative frequency obtained by Donnelly and Walker [12].

\section{THE BINDING POTENTIAL CORRECTION}

The binding potential Eq. (21) can be rewritten, after summing over the spin and isospin of the struck nucleon, and where now $\alpha=0 S, 0 P$, as

$$
\bar{V}_{\alpha}(q)=4\left(2 \ell_{\alpha}+1\right) \int d r r^{2} R_{\alpha}^{2}(r) j_{0}(q r) V_{1}(r)
$$

where $j_{0}(z)$ is the spherical Bessel function. The binding corrections in the $\mathrm{HO}$ model can be evaluated analyti- cally and assume the forms

$$
\bar{V}_{S}(q)=3 \hbar \omega_{0}\left[1-\frac{q^{2}}{6 \gamma^{2}}\right] \exp \left(-q^{2} / 4 \gamma^{2}\right),
$$

for the $0 S$ state and

$$
\bar{V}_{P}(q)=15 \hbar \omega_{0}\left[1-\frac{q^{2}}{3 \gamma^{2}}+\frac{1}{60}\left(\frac{q^{2}}{\gamma^{2}}\right)^{2}\right] \exp \left(-q^{2} / 4 \gamma^{2}\right)
$$

for the $0 P$ state. In these equations, $\gamma^{2}=m \omega_{0} / \hbar$. The corresponding matter densities, normalized to the number of nucleons in each shell, are

$$
\begin{gathered}
\bar{\rho}_{S}(q)=4 \exp \left(-q^{2} / 4 \gamma^{2}\right) \\
\bar{\rho}_{P}(q)=12\left[1-\frac{q^{2}}{6 \gamma^{2}}\right] \exp \left(-q^{2} / 4 \gamma^{2}\right) .
\end{gathered}
$$

It follows that the binding potential corrections $\bar{V}_{\alpha}$ decay more slowly with $q$ than the corresponding matter densities and at sufficiently large momentum transfers the binding potential will dominate the density term. The effective single-particle energies in the $\mathrm{HO}$ case are

$$
\begin{aligned}
& \mathcal{E}_{S}(q)=\epsilon_{S}-\frac{\bar{V}_{S}(q)}{\bar{\rho}_{S}(q)}=\frac{3}{4} \hbar \omega_{0}\left\{2-\left[1-\frac{q^{2}}{6 \gamma^{2}}\right]\right\} \\
& \mathcal{E}_{P}(q)=\epsilon_{P}-\frac{\bar{V}_{P}(q)}{\bar{\rho}_{P}(q)} \\
& =\frac{5}{4} \hbar \omega_{0}\left\{2-\left[1-\frac{q^{2}}{3 \gamma^{2}}\right.\right. \\
& \left.\left.+\frac{1}{60}\left(\frac{q^{2}}{\gamma^{2}}\right)^{2}\right] /\left[1-\frac{q^{2}}{6 \gamma^{2}}\right]\right\} \text {. }
\end{aligned}
$$

In Fig. 2 we show $\mathcal{E}_{\alpha}(q)$ for the $0 S$ and $0 P$ shells when using the Woods-Saxon (solid line) and HO (dashed line) shell-model wave functions. The results for $q=0$ are readily understood. $\bar{V}_{\alpha}(q=0)$ is the expectation value of the binding potential in state $\alpha . \mathcal{E}_{\alpha}(q=0)$ is therefore the average kinetic energy in the state $\alpha$ and is necessarily positive. It can be checked that Eqs. (35) and (36) give the correct oscillator kinetic energies in the $q=0$ limit.

As the momentum transfer increases from zero, the positive energy shift increases, essentially because the factor $V_{1}(r)$ in Eq. (30) makes the mean-square radius of the distribution $R_{\alpha}^{2}(r) V_{1}(r)$ smaller than that of $R_{\alpha}^{2}(r)$. Except near the zero of $\bar{\rho}_{P}(q)$ in the $0 P$ case, the energy shift is positive, as is shown in Fig. 2. The inclusion of the binding energy and binding potential effects thus suggests the use of positive, momentum-transfer-dependent effective single-particle energies.

This result must be contrasted with what is assumed when considering only the binding energy of the struck 

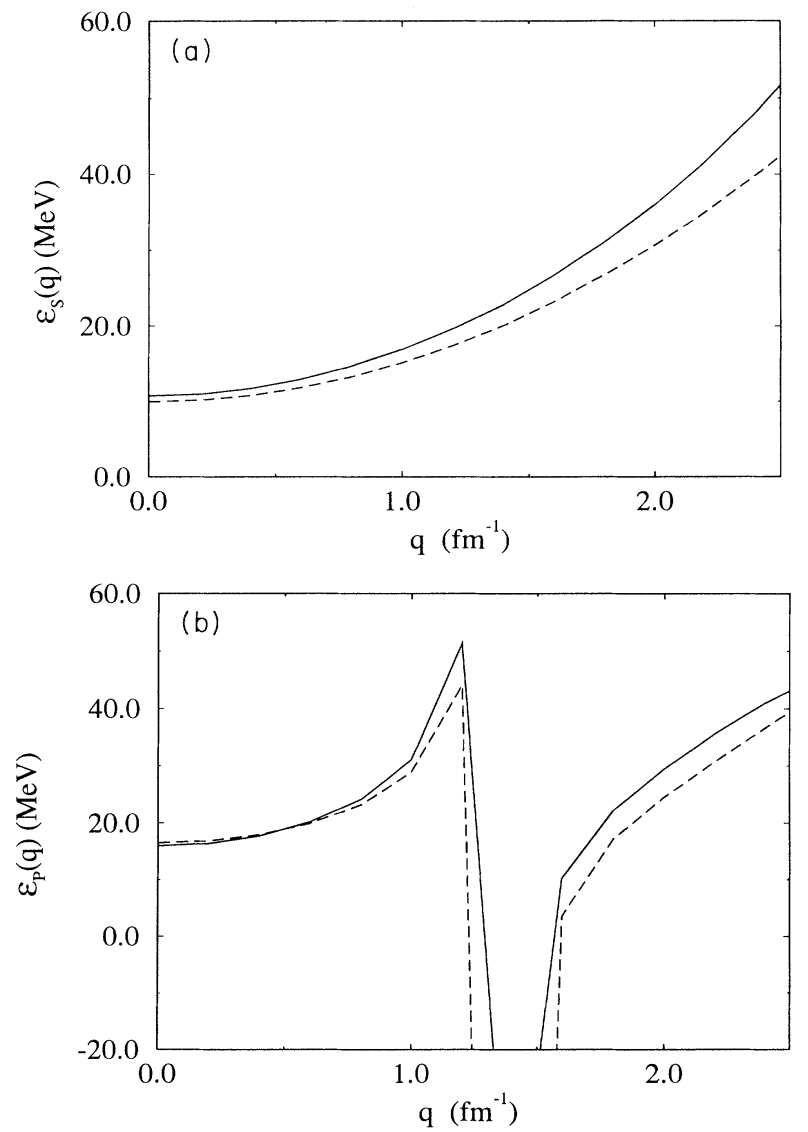

FIG. 2. Effective single-particle energies $\mathcal{E}_{\alpha}(q)$ for the $0 S$ and $0 P$ shell when using Woods-Saxon model (solid line) and $\mathrm{HO}$ model (dashed line) single-particle wave functions.

nucleon, and which results in an average negative energy shift. The value $\langle\epsilon\rangle=-20 \mathrm{MeV}$ was used in the work of Ref. [2]. That work, however, also included a careful treatment of the dependence of the energy parameter of the $N N$ amplitude on the momenta of the interacting $N N$ pair within the full folding framework. The energy used was

$$
\omega_{\alpha}(N N)=E+\langle\epsilon\rangle-\hbar^{2} \mathcal{P}^{2} / 4 m
$$

where $\mathcal{P}$, the total momentum of the interacting nucleons, was treated accurately. Significant from the point of view of the present work, however, is that the inclusion of this $\mathcal{P}$ variation on its own, i.e., with $\langle\epsilon\rangle=0$, produced only relatively minor modifications to the calculated elastic scattering observables. It was found in [2] that only with the inclusion of the negative binding energy correction, $\langle\epsilon\rangle=-20 \mathrm{MeV}$, did the $\mathcal{P}$ variation introduce significant changes to the calculated observables. This suggests that in the absence of this large negative energy offset, the range of the $N N$ energy parameter sampled by the $\mathcal{P}$ variation does not extend to regions in which the $N N$ transition amplitude shows a significant energy dependence.

The present work does not include these $\mathcal{P}$ variations but does include binding potential corrections explicitly.
Our results suggest that the inclusion of the effects of the binding potential lead to positive effective singleparticle energies $\mathcal{E}_{\alpha}(q)$, as opposed to the negative singleparticle energies usually used in first-order KMT calculations. Given the sensitivity of the calculations of [2] referred to above to the inclusion of a negative average single-particle energy, this could alter significantly the conclusions reached in [2] regarding the importance of the nonlocal corrections associated with the momenta of the interacting nucleons; associated with $\mathcal{P}$ in Eq. (37).

We note that the effective energy for the $0 P$ shell exhibits a pole at the zero of $\bar{\rho}_{P}(q)$. However, since in the evaluation of the optical potential the transition amplitude appears multiplied by the density, the correction terms are perfectly well defined and finite. The appropriate cancellations are taken account of automatically when Eq. (26) is used.

\section{THE ELASTIC SCATTERING OBSERVABLES}

The off-shell matrix elements of the $N N$ transition amplitudes are very slowly varying functions of the $N N$ center-of-mass energy [13] at energies of relevance to the optimal factorization calculations. To estimate quantitatively the binding energy and potential corrections we need to evaluate very accurately the energy derivative of the $N N$ transition amplitude off the energy shell, required by Eq. (26). In order to achieve this we use a cubic spline interpolation procedure as developed by Glockle, Hasberg, and Neghabian [14]. The optical potential was evaluated in the momentum-space representation [15].

A simple estimate was made of the order of magnitude of the binding effects in the evaluation of the optical potential. We considered the simple case where the projectile and struck nucleon momenta are fixed at the on-shell value. We calculated in this case that the real part of the optical potential would typically be modified by $2 \%$ and that the effects on the imaginary part were negli-

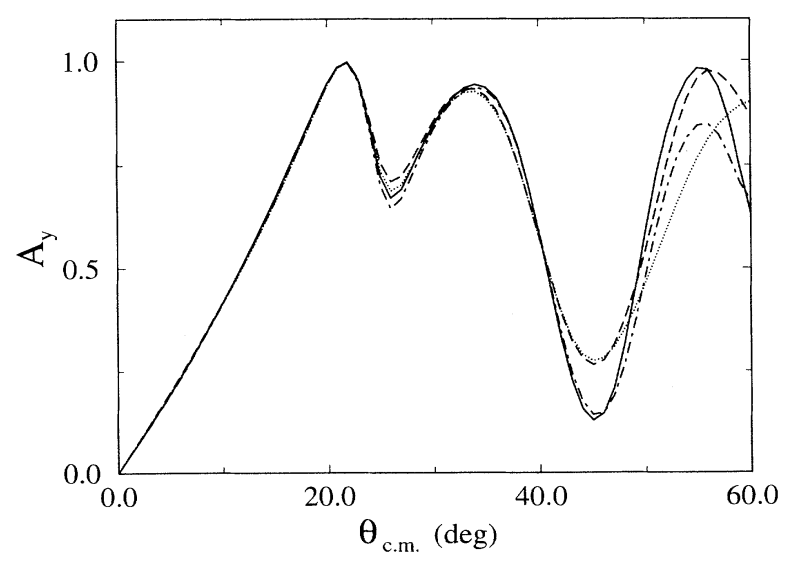

FIG. 3. Analyzing power for ${ }^{16} \mathrm{O}(p, p)$ at $200 \mathrm{MeV}$, predicted by the binding effects with Woods-Saxon (solid line) and $\mathrm{HO}$ (dashed-dotted) radial wave functions. The dashed line and the dotted line show the corresponding curves using the optimal factorization approximation. 
gible. Thus only relatively minor changes are expected in the calculated optical potential and elastic scattering observables.

In Fig. 3 we show the analyzing power for proton nucleus elastic scattering at $200 \mathrm{MeV}$, in the absence of the Coulomb interaction. This observable displays most sensitivity to small changes in the optical potential. The solid (dashed) line represents the analyzing power with (without) the binding corrections as described in the text, using Woods-Saxon radial wave functions. The dasheddotted and dotted lines represent the corresponding calculations using HO radial wave functions. As is evident from the figure, the binding effects are very small, as might be anticipated from the previous simple estimate. Changes in the calculated total reaction cross sections are of order $1 \%$.

\section{CONCLUSIONS}

In this paper we have calculated the medium effects, due to the binding energy and binding potential of the struck nucleon, on the nucleon-nucleus interaction. We have used the off-shell $N N$ transition amplitude derived from the Paris potential, evaluated at a fixed energy. We have shown that the effects of the binding potential, in leading order, can be taken into account by the use of a positive, momentum-transfer-dependent effective singleparticle energy in the first-order KMT optical potential. This is to be contrasted with the constant negative singleparticle energies usually used in first-order KMT calculations. The binding energy and potential corrections to the optical potential were estimated using an expansion about the impulse approximation value and the effects on the elastic scattering observables were found to be small.

The use of a positive effective single-particle energy in the present work may have implications for the conclusions of the analysis of Arellano, Brieva, and Love [2] regarding the importance of those corrections to the impulse approximation associated with the detailed treatment of the momenta of the interacting nucleon pair. The results of the present work effectively shifts the energy parameter in the $N N$ transition amplitude to higher energies whereas the negative shift used in [2] moves the $N N$ energy to smaller values and then varies it according to the momenta of the colliding nucleon pair. When in [2] the negative binding energy shift was removed, but the energy variation due to the momenta of the colliding nucleon pair retained, only small corrections to the impulse approximation result were obtained.

A fully consistent calculation incorporating both mechanisms will be needed to clarify the situation.

\section{ACKNOWLEDGMENTS}

The financial support of the Instituto Nacional de Investigação Científica (INIC) and of the British Council which made the collaboration possible is gratefully acknowledged. The support of Junta Nacional de Investigação Científica e Técnológica (JNICT) through Grant No. 1094/CEN (for R.C.) and of the Science and Engineering Research Council (U.K.) through Grants No. GR/G/0109.6 and No. GR/H/2402.0 (for J.A.T. and R.C.J.) are also acknowledged. We would also like to thank Centro de Matemática e Aplicações Fundamentais for providing computer facilites. The use of the numerical spline interpolation routines of Professor A.C. Fonseca and Dr. E. Cravo is also acknowledged.
[1] A.K. Kerman, H. McManus, and R.M. Thaler, Ann. Phys. (N.Y.) 8, 551 (1959).

[2] H.F. Arellano, F.A. Brieva, and W.G. Love, Phys. Rev. C 41, 2188 (1990); H.F. Arellano, W.G. Love, and F.A. Brieva, ibid. 43, 2734 (1991).

[3] Ch. Elster, T. Cheon, E.F. Redish, and P.C. Tandy, Phys. Rev. C 41, 814 (1990); C.R. Chinn, Ch. Elster, and R.M. Thaler, ibid. 44, 1569 (1991).

[4] R. Crespo, R.C. Johnson, and J.A. Tostevin, Phys. Rev. C 41, 2257 (1990).

[5] R. Crespo, R.C. Johnson, and J.A. Tostevin, Phys. Rev. C 44, R1735 (1991); 46, 279 (1992); R. Crespo, Ph.D. thesis, University of Surrey, 1991 (unpublished).

[6] P.C. Tandy, E.F. Redish, and D. Bollé, Phys. Rev. Lett. 35, 923 (1975).

[7] P.C. Tandy, E.F. Redish, and D. Bollé, Phys. Rev. C 16, 1924 (1977).
[8] A.W. Thomas and R.H. Landau, Phys. Rep. 58, 121 (1980).

[9] L. Ray, Phys. Rev. C 41, 2816 (1990).

[10] M. Lacombe, B. Loiseau, J.M. Richard, R. Vinh Mau, J. Côté, P. Pires, and R. de Tourreil, Phys. Rev. C 21, 861 (1980).

[11] I. Sick and I.S. McCarthy, Nucl. Phys. A150, 631 (1970).

[12] T.W. Donnelly and G.E. Walker, Phys. Rev. Lett. 22, 1121 (1969).

[13] E.F. Redish and K. Stricker-Bauer, Phys. Rev. C 35, 1183 (1987); 36, 513 (1987); E.F. Redish, Nucl. Phys. A463, 417c (1987).

[14] W. Glockle, G. Hasberg, and A.R. Neghabian, Z. Phys. A 305, 217 (1982).

[15] An extension of the program LPOTP, M.J. Paez, M.E. Sagen, and R.H. Landau, Comput. Phys. Commun. 52, 141 (1988). 